Quantitative unconditionality of Banach spaces $E$ for which $\mathscr{K}(E)$ is an $M$-ideal in $\mathscr{L}(E)$

by

DANIEL LI (Paris)

Abstract. Let $E$ be a separable Banach space with AP; if $\mathscr{K}(E)$ is an $M$-ideal in $\mathscr{L}(E)$, then for every $\varepsilon>0, E$ is isometric to a $(1+\varepsilon)$-complemented subspace in a space with a $(1+i)$-unconditional shrinking FDD; in particular, $E$ is isometric to a subspace of a space with a $(1+\varepsilon)$-unconditional basis, and $\operatorname{dist}\left(E, L^{p}(0,1)\right) \geqslant \max (p-1,1 /(p-1))$.

1. Introduction. Since E. Alfsen and E. Effros introduced the notion of an $M$-ideal [1], many authors have studied for which Banach spaces $E, \mathscr{K}(E)$, the space of compact operators on $E$, is an $M$-ideal in $\mathscr{L}(E)$, the space of all continuous operators on $E$. J. Hennefeld [17] had already proved that if $E=c_{0}$ or $l^{p}, 1<p<\infty, \mathscr{K}(E)$ is an $M$-ideal in $\mathscr{L}(E)$; actually for $E=l^{2}$ this had been proved a long time ago by J. Dixmier [8]. This was improved by $\AA$. Lima: if $\left(E_{\alpha}\right)_{\alpha \in A}$ is a family of finite-dimensional spaces and $E=\left(\oplus_{\alpha \in A} E_{\alpha}\right)_{l p}, 1<p<\infty$, or $E=\left(\oplus_{\alpha \in A} E_{\alpha)_{\tau_{0}}}\right.$, then $\mathscr{K}(E)$ is an $M$-ideal in $\mathscr{L}(E)$ ([21], p. 31); moreover, if $F$ is a subspace of $E=\left(\oplus_{\alpha \in A} E_{\alpha}\right\rangle_{p}, 1<p<\infty$, C. M. Cho and W. B. Johnson showed that $\mathscr{K}(F)$ is an $M$-ideal in $\mathscr{L}(F)$ if and only if $F$ has the compact approximation property [5]. On the other hand, $\mathscr{K}(E)$ is not an $M$-ideal in $\mathscr{L}(E)$ when a) $E=l^{1}, E=l^{\infty}[31]$, b) $E$ is a space with a 1 -symmetric basis which is not $l^{p}$ or $c_{0}[18]$, c) $E=\mathscr{C}(K), K$ infinite compact [9], d) $E=L^{1}(\mu)$, $\operatorname{dim} L^{1}(\mu)=\infty$ [9], [20], e) $E$ is a Lindenstrauss space which is not $c_{0}(I)$, f) $E=L^{p}(\mu), 1<p<\infty, p \neq 2, \mu$ not purely atomic [20]. Some authors also studied when $\mathscr{K}(X, Y)$ is an $M$-ideal in $\mathscr{L}(X, Y)$ ([2], [9], [12], [20], [26], for instance).

After this concrete examples, structure theorems were sought for Banach spaces $E$ for which $\mathscr{K}(E)$ is an $M$-ideal in $\mathscr{L}(E)$; $\AA$. Lima proved that $E$ is then an $M$-ideal in its bidual $E^{* *}[21]$, a result which also yields the previous examples a), c), d), e) [16]. Lately G. Godefroy and P. Saab showed that $E$ (supposed separable with AP) has got to be isomorphic to a complemented subspace of a space with a shrinking unconditional finite-dimensional decomposition [15].

1985 Mathematics Subject Classification 46B20, 47D15, 47D30, 46B15, 46B10. 
The present work is a quantitative version of [15]; we prove that if $E$ is a separable Banach space with $\mathrm{AP}$ such that $\mathscr{K}(E)$ is an $M$-ideal in $\mathscr{L}(E)$, then for every $\varepsilon>0, E$ is isometric to a $(1+\varepsilon)$-complemented subspace in a space with a $(1+\varepsilon)$-unconditional shrinking FDD; in particular, $E$ is isometric to a subspace of a space with a $(1+\varepsilon)$-unconditional basis and

$$
\operatorname{dist}\left(E, L^{p}(0,1)\right) \geqslant \max \left(p-1, \frac{1}{p-1}\right) .
$$

This answers a question of G. Godefroy.

Let us note, however, that the main argument of [15] (numerical radius) cannot give this quantitative result and it is replaced by the fundamental lemma 4.1 of E. Alfsen and E. Effros [1].

2. Notations and preliminaries. The Banach spaces considered in this paper are real or complex; all the subspaces are supposed to be closed. If $X$ is a Banach space, its closed unit ball is denoted by $X_{1}$, and the weak*-topology on the dual $X^{*}$ by $\omega^{*}$; in any case, the notations are standard and are those of [7] or [23].

If $E$ is a Banach space, $\mathscr{K}(E)$ denotes the space of compact operators on $E$, $\mathscr{L}(E)$ is the space of all continuous linear operators on $E$, and $\mathscr{F}(E)$ the space of finite rank ones.

A subspace $J$ of a Banach space $X$ is said to be an $M$-ideal if there is an $L$-projection $Q$ of $X^{*}$ with range $J^{\perp}$, the annihilator of $J$ in $X^{*}$, that is, a projection $Q$ such that $\|x\|=\|Q x\|+\|x-Q x\|$ for every $x \in X^{*}$.

A Banach space $F$ is said to have a finite-dimensional Schauder decomposition (FDD) if every $x \in F$ can be uniquely written as $x=\sum_{n=1}^{\infty} x_{n}$, where $x_{n} \in F_{n}$, and each $F_{n}$ is a finite-dimensional subspace of $F$; the FDD is said to be unconditional if the series $\sum_{n=1}^{\infty} x_{n}$ is unconditionally convergent for every $x \in F$,

$$
\sup _{\|x\| \leqslant 1} \sup _{N \geqslant 1} \sup _{\left|\theta_{n}\right| \leqslant 1}\left\|\sum_{n=1}^{N} \theta_{n} x_{n}\right\|
$$

is the unconditional constant of the FDD; the FDD is said to be shrinking if it induces by duality an FDD of $F^{*}$ (see [23], I.1.g).

3. Acknowledgements. This work was begun while I was visiting the University of Missouri-Columbia in March-April 1988. I thank the Ministère des Affaires Etrangères for partial financial support, and $\mathrm{P}$, and $\mathrm{E}$. Saab for their warm welcome. I am specially glad to thank M. and G. Godefroy for their very enjoyable hospitality, and $G$. Godefroy for his kind advice and suggestions.

I thank B. Maurey, F. Piquard and J. Saint Raymond for useful conversations, N. Ghoussoub for the reference [3], and D. Werner for useful remarks.

\section{The structure theorem}

THEOREM. Let $E$ be a separable Banach space. We suppose that

(H) There exist a subalgebra $\mathscr{A}$ of $\mathscr{L}(E)$, containing $I=\mathrm{Id}_{E}$, and an ideal $\mathscr{B}$ of $\mathscr{A}$ such that:

1) $\mathscr{B} \subset \overline{\mathscr{F}(E)}$ (norm closure),

2) $\left(\exists B_{n} \in \mathscr{B}\right)\left\|B_{n}\right\| \leqslant 1,\left\|B_{n} x-x\right\| \rightarrow 0, \forall x \in E$,

3) $\mathscr{B}$ is an $M$-ideal in $\mathscr{A}$.

Then

(C) For every $\varepsilon>0$, there exists a space $F$ with a $(1+\varepsilon)$-unconditional $F D D$ such that $E$ is isometric to a $(1+\varepsilon)$-complemented subspace in $F$. Moreover, if $E \ngtr l^{1}$. (resp. $E$ is reflexive) we can take $F$ such that its $F D D$ is shrinking (resp. $F$ reflexive).

We will prove this theorem in the next section. Let us now present a few consequences.

COROLlary 1. Let $E$ be a separable Banach space satisfying $(\mathrm{H})$. Then

(C) For every $\varepsilon>0, E$ is isometric to a subspace of a space $G$ with $a(1+\varepsilon)$-unconditional basis. Moreover, if $E \neq l^{1}$ (resp. $E$ is reflexive), then the basis may be assumed to be shrinking (resp. G may be assumed reflexive).

Proof. This follows from [23], I.1.g.5.: instead of $\left\|x^{*}-x_{i, n}^{*}\right\| \leqslant 4^{-n}$, we take $\left\|x^{*}-x_{i, n}^{*}\right\| \leqslant 2^{-n} \alpha$; then $\varphi(T u) \geqslant 1-\alpha$ and $\varphi\left(M_{\theta} T x\right) \leqslant 1+\alpha$, so that $\|T u\| \geqslant(1-\alpha) /(1+\alpha)$ and $\|T\|\left\|T^{-1}\right\| \leqslant(1+\alpha) /(1-\alpha)$.

Hence, if $F$ admits a $(1+\alpha)$-unconditional FDD, $F$ is isometric to a subspace of $G$ with a $(1+\alpha)^{2} /(1-\alpha)$-unconditional basis.

We conclude the proof by using the interpolation technique of [11], Theorem 3.3.

COROLlary 2, Let $E$ be separable Banach space with the Approximation Property. If $\mathscr{K}(E)$ is an $M$-ideal in $\mathscr{L}(E)$, then for every $\varepsilon>0, E$ is isometric to $a(1+\varepsilon)$-complemented subspace in a space $F$ with $a(1+\varepsilon)$-unconditional shrinking $F D D$, and $F$ may be assumed reflexive if $E$ is.

Proof. It suffices to remark that condition (H 2) holds ([16], Lemma 5.1), and to use [21], Cor. 2.5, in order to see that $E \ngtr l^{1}$.

CoROLlary 3. If $E$ is a separable Banach space with the Approximation Property and $\mathscr{K}(E)$ is an $M$-ideal in $\mathscr{L}(E)$, then

$$
\operatorname{dist}\left(E, L^{p}(0,1)\right) \geqslant \max \left(p-1, \frac{1}{p-1}\right)
$$


Proof. This follows from the reproducibility of the Haar basis ([22], Th. 4.1, [23], II.2.c.7, II.2.c.8): whenever $L^{p}(0,1), 1<p<\infty$, is isometric to a subspace of a space with a basis $\left(u_{n}\right)_{n \geq 1}$, then for every $\varepsilon>0$, there exists a block basis of $\left(u_{n}\right)_{n \geqslant 1}$ which is $(1+\varepsilon)$-equivalent to the Haar basis. Hence, the distance from $E$ to $L^{p}(0,1), 1<p<\infty$, is at least $k_{p}$, the unconditional constant of the Haar basis of $L^{p}(0,1)$, and $k_{p}=\max (p-1,1 /(p-1))$ ([3], Cor. $1,[4])$.

Corollary 3 is also true for $p=1$ and $p=\infty$ since $\mathscr{K}\left(L^{1}(0,1)\right)$ and $\mathscr{K}\left(L^{\infty}(0,1)\right)$ are isomorphic to no space which is an $M$-ideal in its bidual ([21], Cor. 2.5).

Corollary 4. Let E be a Banach space with a monotone basis $\mathscr{U}=\left(u_{n}\right)_{n \geqslant 1}$. If $\mathscr{K}_{\mathrm{d}}(E, \mathscr{U})$, the space of compact diagonal operators, is an $M$-ideal in $\mathscr{L}_{\mathrm{d}}(E, \mathscr{l l})$, the space of continuous diagonal operators, then (C) holds for $E$.

Remark 1. If $E=l^{1}$ and $\mathscr{U}$ is the canonical basis, $\mathscr{K}_{d}\left(l^{1}\right)$ is isometric to $c_{0}$ and is an $M$-ideal of $\mathscr{L}_{\mathrm{d}}\left(l^{1}\right)$ which is isometric to $l^{\infty}$; but $l^{1}$ cannot be isomorphic to a subspace of a space with a shrinking FDD. So, the condition $E \ngtr l^{1}$ in the conclusion (C) of the Theorem cannot be removed.

Remark 2. If $E=L^{p}(\mathbf{T}), 1<p<\infty$, and $\mathscr{U}=\mathscr{T}$ is the trigonometrical basis $\left([23]\right.$, II.2.c.16), then $\mathscr{L}_{\mathrm{d}}\left(L^{p}(\mathbf{T}), \mathscr{T}\right)=\mathrm{CV}\left(L^{p}(\mathbf{T})\right)$ (resp. $\mathscr{H}_{\mathrm{d}}\left(L^{p}(\mathbf{T}), \mathscr{T}\right)$ $\left.=\mathrm{CV}_{\mathrm{c}}\left(L^{p}(\mathrm{~T})\right)\right)$ is the space of convolution operators (resp. compact convolution operators) on $L^{p}(\mathrm{~T})$; as in Cor. $3, C_{\mathrm{c}}\left(L^{p}(\mathrm{~T})\right)$ is not an $M$-ideal in its bidual $\mathrm{CV}\left(L^{p}(\mathrm{~T})\right)$ (another proof of this which was indicated to me by F. Lust-Piquard adapts the one of $\AA$. Lima, [20], Th. 11).

\section{Proof of the Theorem. First, we show} condition:

Proposition. Let $E, \mathscr{A}$ and $\mathscr{B}$ be as in the Theorem, and assume the $\left(\mathrm{H}^{\prime}\right) \quad \mathscr{B} \subset \mathscr{K}(E)$, and $\mathscr{B}$ is separable,

instead of $(\mathrm{H} 1)$. Let $Q$ be the L-projection of $\mathscr{A}^{*}$ with kernel $\mathscr{B}^{\perp}$. Then, for every $\varepsilon>0$, there exists $S_{n} \in \mathscr{B}, n \geqslant 1$, such that

$$
\begin{gathered}
Q^{*} I=\sum_{n=1}^{\infty} S_{n} \quad\left(\sigma\left(\mathscr{A}^{* *}, \mathscr{A}^{*}\right)\right), \\
\sup _{\left|\theta_{k}\right| \leqslant 1}\left\|\sum_{k=1}^{n} \theta_{k} S_{k}\right\| \leqslant 1+\varepsilon .
\end{gathered}
$$

We need the following lemma, which is essentially known ([30], Th. 2.5).

LEMma 1. With the hypothesis of the Proposition, for every $A \in \mathscr{A}$,

$$
Q^{*} A=\lim _{n \rightarrow \infty} B_{n} A \quad\left(\sigma\left(\mathscr{A ^ { * * }}, \mathscr{A}^{*}\right)\right) .
$$

Proof. We use the argument of J. Johnson ([19], Lemma 1). For every ultrafilter $\mathscr{V} ; \lim _{\mathscr{V}} \psi\left(B_{n}\right)$ exists for every $\psi \in \mathscr{B}^{*}$. $\mathscr{B}$ being an ideal of $\mathscr{A}$, the map $\psi: S \in \mathscr{B} \mapsto \varphi(S A)$ is an element of $\mathscr{B}^{*}$ for every $A \in \mathscr{A}$ and every $\varphi \in \mathscr{B}^{*}$; hence, we can define $\hat{\varphi}(A)=\lim _{\psi} \varphi\left(B_{n} A\right)$; then $\hat{\varphi} \in \mathcal{S}^{*}$, $\|\hat{\varphi}\| \leqslant\|\varphi\|$.

Since $B_{n} \stackrel{\|\cdot\|}{\longrightarrow} I$ as $n \rightarrow \infty$ uniformly on compact subsets of $E$, we have $\left\|B_{n} B-B\right\| \rightarrow 0$ as $n \rightarrow \infty$ for $B \in \mathscr{B}$, and so $\hat{\varphi}(B)=\varphi(B)$ for $B \in \mathscr{B}$; hence $\|\hat{\varphi}\|=\|\varphi\|$.

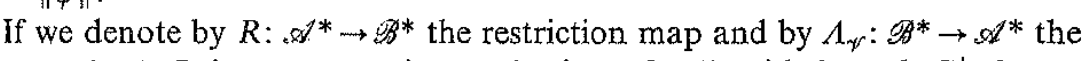
map $\varphi \mapsto \hat{\varphi}, \Lambda_{\mathscr{r}} R$ is a contractive projection of $\mathscr{A}^{*}$ with kernel $\mathscr{B}^{\perp}$; hence $\Lambda_{\mathscr{r}} R=Q$ ([16], Prop. 2.1.b)

Therefore, for $A \in \mathscr{A}$ and $\varphi \in \mathscr{A}^{*}$,

$$
\left\langle Q^{*} A, \varphi\right\rangle=\left\langle A, A_{\mathscr{r}} R \varphi\right\rangle=\lim _{\mathscr{r}}(R \varphi)\left(B_{n} A\right)=\lim _{\mathscr{V}} \varphi\left(B_{n} A\right),
$$

that is, $Q^{*} A=\lim _{n \rightarrow \infty} B_{n} A\left(\sigma\left(\mathscr{A}^{* *}, \mathscr{A}^{*}\right)\right)$.

Proof of the Proposition. Let us consider $\mathscr{A}$ with its real Banach space structure. By Lemma $1, Q^{*} I=\lim _{n \rightarrow \infty} B_{n}\left(\sigma\left(\mathscr{A}^{* *}, \mathscr{A}^{*}\right)\right)$.

Let $K$ be the compact space $\left(\mathscr{A}_{1}^{*}, \omega^{*}\right)$, and let us consider elements of $\mathscr{A}^{* *}$ as functions on $K$. Denoting by $\chi_{L}$ the characteristic function of the set $L \subset K$, we set

$$
h=\left(I \chi_{\mathscr{B} \perp \cap K}\right) \vee 0=\left(I \chi_{\mathscr{B S} \perp \cap K}\right)^{+}
$$

and let $\hat{h}$ be the smallest concave upper-semicontinuous (u.s.c.) function which is greater than $h$ on $K$; then ([1], Lemma 4.1)

$$
(\forall t \in K) \quad\left(I-Q^{*} I\right)(t)=h(t)-h(-t) .
$$

Therefore, setting

$$
(\forall t \in K) \quad g(t)=\frac{1}{2}[I(t)+1]-\widehat{h}(t)
$$

we have

$$
(\forall t \in K) \quad I(Q t)=\left(Q^{*} I\right)(t)=g(t)-g(-t),
$$

and $g$ is a positive, lower-semicontinuous (1.s.c.) function on $K$ such that

$$
(\forall t \in K) \quad g(t)+g(-t) \leqslant 1
$$

I am indebted to J. Saint Raymond for the next lemma.

LeMMA 2. Let $K$ be a compact space and $F: K \rightarrow \mathbf{R}$ such that $F=G_{1}-G_{2}$, with $G_{1}, G_{2}$ positive l.s.c. functions on $K$. If there exist a metrizable compact space $H$, a continuous surjection $R: K \rightarrow H$ and a function $F^{\prime}: H \rightarrow \mathbf{R}$ such that 
$F=F^{\prime} \circ R$, then there exist continuous functions $f_{n}(t)$ on $K, n=1,2, \ldots$, such that

$$
\begin{gathered}
(\forall t \in K) \quad F(t)=\sum_{n=1}^{\infty} f_{n}(t), \\
\sup _{\left|\theta_{n}\right| \leqslant 1}\left\|\sum_{n=1}^{N} \theta_{n} f_{n}\right\|_{\infty} \leqslant \sup _{t \in K}\left[G_{1}(t)+G_{2}(t)\right] .
\end{gathered}
$$

Sketch of proof (see [14], Lemma 3). We set

$$
(\forall u \in H) \quad \tilde{G}_{i}(u)=\inf \left\{G_{i}(t) \mid R(t)=u\right\} ;
$$

then $\tilde{G}_{1}, \tilde{G}_{2}$ are positive and 1.s.c. on $H$, and

$$
(\forall u \in H) \quad F^{\prime}(u)=\tilde{G}_{1}(u)-\tilde{G}_{2}(u) .
$$

Since $H$ is a metrizable space we have

$$
\tilde{G}_{i}(u)=\lim _{n \rightarrow \infty} \uparrow g_{i, n}(u)=\sum_{n=1}^{\infty}\left[g_{i, n+1}(u)-g_{i, n}(u)\right],
$$

with $g_{1,1}=g_{2,1}=0, g_{1, n}, g_{2, n}, n \geqslant 1$, continuous on $H$; setting, for $n \geqslant 1$,

$$
(\forall u \in H) \quad f_{n}^{\prime}(u)=\left[g_{1, n+1}(u)-g_{1, n}(u)\right]-\left[g_{2, n+1}(u)-g_{2, n}(u)\right],
$$

we have

$$
(\forall u \in H) \quad F_{n}^{\prime}(u)=\sum_{n=1}^{\infty} f_{n}^{\prime}(u)
$$

and, for $\left|\theta_{n}\right| \leqslant 1, u \in H$,

$$
\begin{aligned}
\left|\sum_{n=1}^{N} \theta_{n} f_{n}^{\prime}(u)\right| & \leqslant \sum_{n=1}^{\infty}\left[g_{1, n+1}(u)-g_{1, n}(u)\right]+\sum_{n=1}^{\infty}\left[g_{2, n+1}(u)-g_{2, n}(u)\right] \\
& =\tilde{G}_{1}(u)+\tilde{G}_{2}(u) \leqslant \sup _{t \in K}\left[G_{1}(t)+G_{2}(t)\right] .
\end{aligned}
$$

This gives the lemma with $f_{n}=f_{n}^{\prime} \circ R$.

We use Lemma 2 with $K=\left(\mathscr{A}_{1}^{*}, \omega^{*}\right), H=\left(\mathscr{B}_{1}^{*}, \omega^{*}\right), F=Q^{*} I$, and $R$ the restriction map. Since $Q^{*} I=F^{\prime} \circ R$, where

$$
F^{\prime}(u)=\hat{u}(I)=\lim _{r} u\left(B_{n}\right)=\lim _{k \rightarrow \infty} u\left(B_{n_{k}}\right)
$$

we get by Lemma 2 functions $f_{n} \in \mathscr{C}(K)$ such that

$$
(\forall t \in K) \quad\left(Q^{*} I\right)(t)=\sum_{n=1}^{\infty} f_{n}(t),
$$

$\sup _{\left|\theta_{n}\right| \leqslant 1}\left\|\sum_{n=1}^{N} \theta_{n} f_{n}\right\|_{\infty} \leqslant \sup _{t \in K}[g(t)+g(-t)] \leqslant 1$.
In order to conclude the proof of the Proposition, it suffices to apply the following quantitative version of a classical result of A. Pełczyński (a similar result can be found in [10], Lemma 1), with $Y=\mathscr{B}, X=\mathscr{C}(K)$.

LEMMA 3. Let $X$ be a Banach space and let $Y$ be a subspace of $X$. If $f \in Y^{\perp \perp}$ is such that

$$
\begin{gathered}
f=\sum_{n=1}^{\infty} \omega^{*} x_{n}, \quad x_{n} \in X, \\
\sup _{\left|\theta_{n}\right| \leqslant 1}\left\|\sum_{n=1}^{N} \theta_{n} x_{n}\right\| \leqslant C\|f\|,
\end{gathered}
$$

then, for every $\varepsilon>0$, there are $y_{n} \in Y, n \geqslant 1$, such that

$$
f=\sum_{n=1}^{\infty} \omega^{*} y_{n}, \quad \sup _{\left|\theta_{n}\right| \leqslant 1}\left\|\sum_{n=1}^{N} \theta_{n} y_{n}\right\| \leqslant(C+\varepsilon)\|f\| .
$$

Sketch of proof (see [29], Lemma 15.7, p. 446). There exist $z_{n} \in Y$, $n \geqslant 1$, such that $f=\omega^{*}-\lim _{n \rightarrow \infty} z_{n}$. Setting $v_{n}=z_{n}-\sum_{i=1}^{n} x_{i}, n \geqslant 1$, we can define a strictly increasing sequence of integers $0=p_{0}<p_{1}<\ldots$, a sequence of real numbers $\lambda_{n} \geqslant 0$, such that $\sum_{i=p_{n-1}+1}^{p_{n}} \lambda_{i}=1$ and

$$
u_{n}=\sum_{i=p_{n-1}+1}^{p_{n}} \lambda_{i} v_{i}, \quad\left\|u_{n}\right\| \leqslant \varepsilon\|f\| / 2^{n+1} .
$$

Setting now

$$
\begin{aligned}
& w_{n}=\sum_{i=p_{n-1}+1}^{p_{n}} \lambda_{i} z_{i}, \\
& y_{1}=w_{1}, \quad y_{n+1}=w_{n+1}-w_{n},
\end{aligned}
$$

we have

$$
\begin{aligned}
y_{1} & =\sum_{j=1}^{p_{1}} \mu_{j}^{0} x_{j}+u_{1}, \\
y_{n+1} & =\sum_{j=p_{n-1}+1}^{p_{n+1}} \mu_{j}^{n} x_{j}+u_{n+1}-u_{n}, \quad n \geqslant 1,
\end{aligned}
$$

with

$$
\begin{aligned}
& \mu_{j}^{0}=\sum_{i=j}^{p_{1}} \lambda_{i} . \\
& \mu_{j}^{n}= \begin{cases}1-\sum_{i=j}^{p_{n}} \lambda_{i}, & p_{n-1}+1 \leqslant j \leqslant p_{n}, \\
\sum_{i=j}^{p_{n+1}} \lambda_{i}, & p_{n}+1 \leqslant j \leqslant p_{n+1},\end{cases}
\end{aligned}
$$


Since $0 \leqslant \mu_{j}^{n} \leqslant 1, \forall j, \forall n \geqslant 0$, we obtain

$$
\begin{aligned}
& \left\|\sum_{n=1}^{N} \theta_{n} y_{n}\right\| \\
& \leqslant\left\|\sum_{n=1}^{N-1} \sum_{j=p_{n-1}+1}^{p_{n}}\left(\theta_{n} \mu_{j}^{n-1}+\theta_{n+1} \mu_{j}^{n}\right) x_{j}+\sum_{j=p_{N-1}+1}^{p_{N}} \theta_{N} \mu_{j}^{N-1} x_{j}\right\|+\varepsilon\|f\| \\
& \leqslant C\|f\|+\varepsilon\|f\|
\end{aligned}
$$

because $\mu_{j}^{n-1}+\mu_{j}^{n}=1$ for $p_{n-1}+1 \leqslant j \leqslant p_{n}$, and hence $\left|0_{n} \mu_{j}^{n-1}+0_{n+1} \mu_{j}^{n}\right| \leqslant 1$.

The conclusion of the proof of the Theorem is along the lines of [15].

We begin by noting that, for $x \in E, x^{*} \in E^{*}, x \otimes x^{*}$ induces an element of $\mathscr{A}^{*}$ :

$$
(\forall A \in \mathscr{A}) \quad\left\langle x \otimes x^{*}, A\right\rangle=\left\langle x^{*}, A x\right\rangle
$$

we have $\left\|x \otimes x^{*}\right\|_{\mathscr{A}^{*}} \geqslant\left\|R\left(x \otimes x^{*}\right)\right\|_{\text {络* }}$; on the other hand, for every $A \in \mathscr{A}$, $B_{n} A \in \mathscr{B}$ and condition (H2) gives

$$
\left\langle x^{*}, A x\right\rangle=\lim _{n \rightarrow \infty}\left\langle x^{*}, B_{n} A x\right\rangle ;
$$

hence $\left\|x \otimes x^{*}\right\|_{\mathscr{A}^{*}}=\left\|R\left(x \otimes x^{*}\right)\right\|_{\mathscr{B}^{*}}$.

Therefore, since $\mathscr{A}^{*}=\mathscr{B}^{\perp} \oplus_{1} N$ with $N$ isometrically isomorphic to $\mathscr{B}^{*}$, we have $Q\left(x \otimes x^{*}\right)=x \otimes x^{*}$, and the Proposition allows us to write

$$
\left\langle x^{*}, x\right\rangle=\sum_{n=1}^{\infty}\left\langle x^{*}, S_{n} x\right\rangle .
$$

By condition (H1), we can find a sequence $\left(R_{n}\right)_{n \geqslant 1}$ of finite rank operators such that $\left\|S_{n}-R_{n}\right\| \leqslant \varepsilon / 2^{n}, n \geqslant 1$; setting (as in [27], proof of Prop. 3, or [15], proof of Cor. 6)

$$
(\forall x \in E) \quad S(x)=\sum_{n=1}^{\infty} \omega^{*} R_{n}(x)
$$

we obtain an operator from $E$ into $E^{* *} ; S$ is actually an operator from $E$ into $E$ because for every $x \in E$ and $n \geqslant 1$ we have

$$
\begin{aligned}
\operatorname{dist}(S(x), E) & \leqslant\left\|S(x)-\sum_{k=1}^{n} R_{k}(x)-x+\sum_{k=1}^{n} S_{k}(x)\right\| \\
& \leqslant \sum_{k=n+1}^{\infty}\left\|R_{k}-S_{k}\right\|\|x\| \leqslant \varepsilon\|x\| / 2^{n}
\end{aligned}
$$

moreover, since $\|I-S\| \leqslant \varepsilon, S$ is an invertible operator and $\left\|S^{-1}\right\| \leqslant 1 /(1-\varepsilon)$.
Now, the finite rank operators $U_{n}=S^{-1} R_{n}$ satisfy the conditions

$$
x=\sum_{n=1}^{\infty} U_{n}(x), \quad \sup _{\left|\theta_{n}\right| \leqslant 1}\left\|\sum_{n=1}^{N} \theta_{n} U_{n}(x)\right\| \leqslant \frac{1+2 \varepsilon}{1-\varepsilon}\|x\|, \quad \forall x \in E .
$$

It follows that $\left(U_{n}\right)_{n \geqslant 1}$ is a $\left(\frac{1+2 \varepsilon}{1-\varepsilon}\right)$-unconditional finite-dimensional expansion of identity of $E$

Now, by [24], Lemma 1.2, there is a Banach space $F$ with a 1-unconditional FDD such that $E$ is $\left(\frac{1+2 \varepsilon}{1-\varepsilon}\right)$-isomorphic to a $\left(\frac{1+2 \varepsilon}{1-\varepsilon}\right)$-complemented subspace in $F$. By renorming $F$, we deduce that $F$ has a $\left(\frac{1+2 \varepsilon}{1-\varepsilon}\right)$-unconditional FDD and that $E$ is isometric to a $\left(\frac{1+2 \varepsilon}{1-\varepsilon}\right)^{2}$-complemented subspace of $F$.

In order to complete the proof let $F$ be a Banach space with a $(1+\varepsilon)$ unconditional FDD, $\left(P_{n}\right)_{n} \geqslant 1$ the associated projections, $J$ an isometry from the Banach space $E$ into $F$, and $P$ a projection of $F$ with range $J(E)$ and $\|P\| \leqslant 1+\varepsilon$

As in [15], adapting the proof of [11], Th. 3.3, we set

$$
W=\overline{\operatorname{conv}}\left(\bigcup_{N \geqslant 1}\left\{\left(\sum_{n=1}^{N} \theta_{n} P_{n}\right)\left(J E_{1}\right) ;\left|\theta_{n}\right| \leqslant 1\right\}\right)
$$

The set $W$ is weakly conditionally compact (w.c.c.) if $E \nsubseteq l^{1}$ (resp. $W$ is weakly compact (w.c.) if $E$ is reflexive) ([11], Lemma 3.2); in particular, every sequence in $W$ has a weakly Cauchy subsequence. Define ([6], Lemma 1)

$$
W_{n}=\frac{1}{2}\left(2^{n} W+2^{-n} F_{1}\right), \quad n \geqslant 0,
$$

and $\|x\|=\left(\sum_{n=0}^{\infty}\|x\|_{n}^{2}\right)^{1 / 2}$, where $\|\cdot\|_{n}$ is the gauge of $W_{n}$; then $F_{0}=\{x \in F \mid$ $\|x\|<\infty\}$ is a Banach space and the canonical injection $j: F_{0} \rightarrow F$ is continuous $\left([6]\right.$, Lemma 1 (ii)). Since $W \subset(1+\varepsilon) F_{1}$, it follows that $\|j\| \leqslant 1+\varepsilon$ because $\|x \mid\| \leqslant 1 \Rightarrow\|x\|_{0} \leqslant 1 \Rightarrow x \in \frac{1}{2}\left(W+F_{1}\right) \subset(1+\varepsilon) F_{1}$.

Since $J E_{1} \subset W$, we have $J=j \circ A$, where $A: E \rightarrow F_{0}$ is an isomorphic embedding, with $\|A\| \leqslant 1,\left\|A^{-1}\right\| \leqslant 1+\varepsilon$.

Since $P_{n}(W) \subset W, n \geqslant 1, F_{0}$ has a $(1+\varepsilon)$-unconditional FDD $([6]$, Lemma 1 (viii), (ix)) which is shrinking (resp. $F_{0}$ is reflexive) since $\left(F_{0}\right)_{1}$ $=\bigcap_{n} \geqslant 0 \frac{1}{2}\left(2^{n} W+2^{-n} F_{1}\right)$ is w.c.c. (resp. w.c.) by a classical lemma of Grothendieck ([7], pp. 227, 237).

Renorming $F_{0}$ concludes the proof:

\section{Concluding remarks and questions}

6.1. The Lorentz sequence spaces $d(w, p), 1<p<\infty$, are reflexive Banach spaces with 1 -symmetric basis such that $\mathscr{K}(d(w, p))$ is not an $M$-ideal in $\mathscr{L}(d(w, p))[18]$; therefore the Theorem of this paper gives a necessary 
condition on $E$ for $\mathscr{K}(E)$ to be an $M$-ideal in $\mathscr{L}(E)$ but it is not a sufficient condition. This leads to

QUESTION 1. Is it possible to find a necessary and sufficient condition on $E$ for $\mathscr{K}(E)$ to be an $M$-ideal in $\mathscr{L}(E)$ ?

6.2. Question 2. Can $\mathscr{K}\left(L^{p}(0,1)\right), 1<p<\infty, p \neq 2$, be isomorphic to a space which is an $M$-ideal in its bidual? (see [14], Question III.4).

6.3. Let $E$ be a Banach space with a monotone basis $\mathscr{U}=\left(u_{n}\right)_{n \geqslant 1}$, and let $\bar{u}_{n} \in \mathscr{K}_{\mathrm{d}}(E, \mathscr{U})$ be defined by $\bar{u}_{n}\left(u_{k}\right)=u_{n}$ if $k=n$, and 0 if $k \neq n ;\left(\bar{u}_{n}\right)_{n \geqslant 1}$ is a basis of $\mathscr{K}_{d}(E, \mathscr{U})([28]$, Lemma 5$)$, and it is easy to see that it is equivalent to the canonical basis of $c_{0}$ if and only if $\mathscr{U}$ is unconditional. A. Sersouri ([28], Problème 15$)$ asked if $\mathscr{U}$ is unconditional whenever $\mathscr{K}_{4}(E, \mathscr{U})$ is isomorphic to $c_{0}$. We may ask: is $\mathscr{U}$ unconditional whenever $\mathscr{K}_{\mathrm{d}}(E, \mathscr{U})$ is an $M$-ideal in $\mathscr{L}_{\mathrm{d}}(E, \mathscr{U})$ (resp. $\mathscr{K}_{\mathrm{d}}(E, \mathscr{U})$ is isomorphic to a space which is an $M$-ideal in its bidual)? (Let us remark that $\mathscr{K}_{\mathrm{d}}\left(E, \mathscr{U}^{*}\right)^{* *}=\mathscr{L}_{\mathrm{d}}(E, \mathscr{U})$ if $\left(\bar{u}_{n}\right)_{n \geqslant 1}$ is shrinking, and, in particular, if $\mathscr{U}$ is shrinking or boundedly complete ([28], Th. 8).) The answer is negative: if $\mathscr{U}$ is a conditional basis of $l^{p}, 1<p<\infty \quad\left([23]\right.$, I.2.b.11, II, p. 162), then $\mathscr{K}_{\mathrm{d}}\left(l^{p}, \mathscr{U}\right)$ is an $M$-ideal in $\mathscr{L}_{\mathrm{d}}\left(l^{p}, \mathscr{U}\right)=\mathscr{K}_{\mathrm{d}}\left(l^{p}, \mathscr{U}\right)^{* *}$ since $\mathscr{K}\left(p^{p}\right)$ is an $M$-ideal in its bidual A weaker question can be asked (a partial answer is given in [28], Prop. 16):

Questron 3. If $\mathscr{K}_{\mathrm{d}}(E, \mathscr{U})$ is an $M$-ideal in $\mathscr{L}_{\mathrm{d}}(E, \mathscr{U})$ (resp. $\mathscr{K}_{\mathrm{d}}(E, \mathscr{U})$ is isomorphic to a space which is an $M$-ideal in its bidual), does $\mathscr{U}$ have an unconditional basic subsequence?

For $E=L^{p}(\mathrm{~T}), \mathscr{U}=\mathscr{T}$ the trigonometric basis, the existence of $\Lambda(r)$-sets $\left(r \geqslant \max \left(p, p^{*}\right)\right)$ implies the existence of an unconditional basic subsequence without the $M$-ideal hypothesis ([25], Th. 2.11).

6.4. Let us mention that every separable $\mathscr{L}^{\infty}$-space which is isomorphic to a space which is an $M$-ideal in its bidual is isomorphic to $c_{0}$ [13].

6.5. If we replace condition $(\mathrm{H} 1)$ in the Theorem by the stronger condition:

$\left(H 1^{\prime \prime}\right) \quad \mathscr{F}(E) \cap \mathscr{B}$ is norm dense in $\mathscr{B}$

(which holds if $\mathscr{B}=\mathscr{K}_{\mathrm{d}}(E, \mathscr{U})$ ), we can take in the proof $R_{\mathrm{n}} \in \mathscr{F}(E) \cap \mathscr{B}$ and if $\mathscr{A}$ is closed in the strong operator topology, then $S \in \mathscr{A}$; hence if $\mathscr{A}$ is also a full subalgebra $\mathscr{L}(E)$, then $S^{-1} \in \mathscr{A}$ (these two conditions hold when $\mathscr{A}=\mathscr{L}_{\mathrm{d}}(E, \mathscr{U})$ ); therefore $U_{n} \in \mathscr{F}(E) \cap \mathscr{A}: E$ has a $(1+\varepsilon)$-unconditional finite-dimensional expansion of its identity, $\left(U_{n}\right)_{n \geqslant 1}$, with the $U_{n}$ in $\mathscr{B}$.

\section{References}

[1] E. Alfsen and E. Effros, Structure in real Banach spaces, I, Ann. of Math. 96 (1972), 98-128. [2] E. Behrends, Sur les M-idéaux des espaces d'opérateurs compacts, Publ. Matls. Univ. Paris VII, Sémin. d'Anal. Fonet. Paris VI-VII, 1984-85, 11-18.

[3] D. L. Burkholder, A nonlinear partial differential equation and the unconditional constant of the Huar system in $L^{n}$. Bull. Amer. Math. Soc. 7 (3) (1982), 591 195.

[4] -, A pronf of Pelczynski's conjecture for the Haar system, Studia Math. 91 (1) (1988), 79-83. 5] C.-M. Cho and W. B. Johnson, A characterization of subspaces $X$ of $l_{n}$ for which $K(X)$ is an $M$-ideal in $L(X)$, Proc. Amer. Math. Soc. 93 (3) (1985), 466-470.

[6] J. Davis, T. Figiel, W. B. Johnson and A. Pelezyriski, Factoring weakly compact operators, J. Funct. Anal. 17 (1974), 311-317.

[7] J. Diestel, Sequences and Series in Banach Spaces, Springer, 1984.

[8] J. Dixmier, Les fonctionnelles linéaires sur l'ensemble des opérateurs bornés d'un espace de Hilhert, Ann. of Math. 51 (1950), 387-408.

[9] H. Fal h hour y, Sur les M-idéaux dans certains espaces d'opérateurs et l'approximation par des opérateurs compacts, Canad. Math. Bull. 23 (4) (1980), 401-411.

[10] M. Fed er, On subspaces of spaces with an unconditional basis and spaces of operators, Illinois J. Math. 24 (2) (1980), 196-205.

[11] T. Figiel, W. B. Johnson and L. Tzafriri, On Banach lattices and spaces having local unconditional structure, with applications to Lorentz function spaces, J. Approx. Theory 13 (1975), 395-412

[12] D. J. Fleming and D. M. Giarrusso, M-ideals in $L\left(l_{1}, E\right)$, Canad. Math. Bull. 29 (1) (1986), 310.

[13] G. Godefroy, $\mathscr{L}^{x}$-Spaces which are isomorphic to M-ideals, preprint.

[14] G. Godefroy and D. Li, Banach spaces which are M-ideals in their bidual have property (u), Ann. Inst. Fourier (Grenoble) 39 (2) (1989), 361 371.

[15] G. Godefroy and P. Sa ab, Weakly unconditionally convergent series in M-ideals, Math. Scand., to appear.

[16] P. Harmand and $\AA$. Lima, Banach spaces which are $M$-ideals in their biduals, Trans. Amer. Math. Soc. 283 (1984), 253-264.

[17] J. Hennofeld, $A$ decomposition for $B(X)^{*}$ and unigue Hahn-Banach extensions, Pacific J. Math. 46 (1) (1973), 197-199.

[18] -, M-ideals, HB-subspaces, and compact operators, Indiana Univ. Math. J. 28 (6) (1979), 927-934.

[19] J. Johnson, Remarks on Banach spaces of compact operators, J. Funct. Anal. 32 (1979), 304-311.

[20] Å. Lim a, M-ideals of compact operalors in classical Banach spaces, Math. Scand. 44 (1979), $207-217$.

[21] -, On M-idcals and best approximation, Indiana Univ. Math, J. 31 (1982), 27-36.

[22] J. Lindenstrauss and A. Pełczyński, Coniributions to the theory of the classical Banach spaces, J. Funct. Anal. 8 (1971), 225 249.

[23] J. Lindenstrauss and L. Tzafriri, Classical Banach Spaces I, II, Springer, 1977, 1979.

[24] A. Pelczyniski and P. Wojtaszczyk, Banach spaces with finite dimensional expansions of identity and universal bases of finite dimensional subspaces, Studia Math. 40 (1971), 91-108.

[25] H. P. Rosenthal, Projections onto translation-invariant subspaces of $L^{P}(G)$, Mem. Amer. Math. Soc. 63 (1966).

[26] K. Saatkamp, M-ideals of compact operators, Math. Z. 158 (1978), 253-263.

[27] A. Sersouri, Propriêté ( $u$ ) dans les espaces d'opérateurs, Bull. Polish Acad. Sci., to appear.

[28] -, Operateurs diagonaux dans les espaces à bases, Math. Z., to appear.

[29] I, Singer, Bases in Banach Spaces I, Springer, 1970. 
[30] R. R. Simith and J. D. Ward, Applications of convexity and M-ideal theory to quotient Banach algebras, Quart. J. Math. Oxford (2) 30 (1979), 365-384.

[31] -, -, M-ideal structure in Banach algebras, J. Funct. Anal. 27 (1978), 337 349.

FQUIPE D'ANALYSE

U.A. No. 754 au C.N.R.S.

DÉPARTEMENT DE MATHESMATIQUES UNIVERSTT'S PARIS-SUD Tour 46, 4̇ème ètalge

4, P!. Jussieu, 75252 Paris Cedex 05, France Bâl. 425
91405 Orsay Cedex, France

Received October 10,1988 Revised version February 22, 198

\section{Partial differential operators of infinite order with constant coefficients \\ on the space of analytic functions on the polydisc}

SIEGFRIED MOMM (Düsseldorf)

Added in proof (January 1990). D. Werner (Remarks on M-ideals of compact operators, to appear in Quart. J. Math. Oxford) proved that the result of C.-M. Cho and W. B. Johnson also holds for subspaces of $c_{0}$-sums of finite-dimensional spaces. A similar , result is given by $\mathrm{E}$. Oja (C. R. Acad. Sci. Paris 309 (1989), 983-968).

Very recently, P. G. Casazza and N. J. Kalton (Notes on approximation properties in separable Banach spaces, preprint) have introduced the notion of $\mu$-ideal which is more general than that of $M$-ideal and have given a characterization of separable reflexive Banach spaces $X$ with AP for which $\mathscr{K}(X)$ is a $\mu$-ideal in $\mathscr{L}(X)$ some technical conditions we consider the (DFN)-space $A_{P, 1}\left(C^{N}\right):=\left\{f \in A\left(C^{N}\right) \mid \exists k \in N\right.$ : $\| n$ $=\sup _{x \in C^{N}}\left|\int(z)\right| e^{-(1-1 ; k) p(z)}\langle x\}$ of analytic functions on $C^{N}$. If we put $A^{0}\left(C^{N}\right):=\{F \mid \forall k \in N$ : $\left.F^{k} \in A_{n, 1}\left(C^{N}\right)\right\}$ then $A_{0,1}\left(C^{N}\right)$ is an $A_{0}^{\prime}\left(C^{N}\right)$-module such that $F \cdot A_{n, 1}\left(C^{N}\right)$ is a closed subspace of $A_{p, 1}\left(\mathrm{C}^{N}\right)$ for each $F \in A_{p}^{0}\left(C^{N}\right)$. We prove that $F \cdot A_{n, 1}\left(C^{N}\right)$ is a complemented subspace of $A,\left(C^{N}\right)$ for each $F \in A_{n}^{0}\left(\mathrm{C}^{N}\right)$ iff the strong dual $A_{p .1}\left(\mathrm{C}^{N}\right)_{b}^{\prime}$ has the linear topological invariant (DN) if for each $F \in A_{p}^{0}\left(C^{N}\right)$ iff the strong dual $A_{p, 1}\left(C^{N}\right)_{b}^{\prime}$, has the linear topological invariant (DN) iff
$A_{p, 1}\left(C^{N}\right)$ itself is a complemented subspace of a corresponding weighted (LB)-space $L_{z i}^{2},\left(C^{N}\right)$ of locally square integrable functions on $C^{N}$. Applying this result to the function $p(z)-\sum_{i}^{*}, 1 ; z_{i}$ $z \in \mathbb{C}^{N}$, we deduce that each nonzero linear partial differential operator of infinite order with constant coefficients on the Fréchct space $A(\Delta)$ of all analytic functions on the unit polydise $A$ in $C^{N}$ admits a continuous linear right inverse. In our approach we use a sequence space representation of $A_{p, 1}\left(\mathbf{C}^{N}\right)_{b}^{\prime}$ and elementary function theory to give all the projections by explicit cormulas.

For a plurisubharmonic function $p$ on $C^{N}$ denote by $A_{p, 1}\left(\mathbb{C}^{N}\right)$ the space of all entire functions $f$ satisfying $|f(z)| \leqslant A e^{B p(z)}$ for some $A>0,1>B>0$ depending on $f$. If we allow arbitrarily large $B>0$, then we get an algebra denoted by $A_{p}\left(\mathbb{C}^{N}\right)$. Both spaces, endowed with their natural inductive limit topology, are (DFN)-spaces, provided that $p$ satisfies some technical conditions.

Recently Meise and Taylor ([12], [13]) showed for radial weights $p$ that each principal ideal of $A_{p}\left(C^{N}\right)$ is complemented if and only if the strong dual $A_{p}\left(\mathbb{C}^{N}\right)_{b}^{\prime}$ has the linear topological invariant $(\mathrm{DN})$.

In the present paper we find for componentwise radial weights. $p$ that each subspace $F \cdot A_{p, 1}\left(C^{N}\right)$ of $A_{p, 1}\left(C^{N}\right)$ is complemented if and only if the strong dual $A_{p, 1}\left(C^{N}\right)_{\mathrm{b}}^{\prime}$ has the linear topological invariant (DN) (see Wagner [21] and Vogt [19]). Our proof also gives rise to a new and more elementary proof of the above-mentioned result of Meise and Taylor.

To state an application of our result, we denote by $A(\Delta)$ the Fréchet space of all analytic functions on the unit polydisc $\Delta$ in $C^{N}$. Then each nonzero 